\title{
Correlated Dirac fermions on the honeycomb lattice studied within cluster dynamical mean field theory
}

\author{
Ansgar Liebsch \\ Institut für Festkörperforschung, Forschungszentrum Jülich, D-52425 Jülich, Germany
}

(Received 22 October 2010; published 18 January 2011)

\begin{abstract}
The role of nonlocal Coulomb correlations in the honeycomb lattice is investigated within cluster dynamical mean field theory combined with finite-temperature exact diagonalization. The paramagnetic semimetal-toinsulator transition is found to be in excellent agreement with finite-size determinantal quantum Monte Carlo simulations and with cluster dynamical mean field calculations based on the continuous-time quantum Monte Carlo approach. As expected, the critical Coulomb energy is much lower than within a local or single-site formulation. Short-range correlations are shown to give rise to a pseudogap and concomitant non-Fermi-liquid behavior within a narrow range below the Mott transition.
\end{abstract}

DOI: 10.1103/PhysRevB.83.035113

PACS number(s): 71.10.-w, 73.22.-f

\section{INTRODUCTION}

The recent discovery of graphene ${ }^{1}$ has greatly stimulated the study of the electronic properties of the honeycomb lattice. ${ }^{2}$ In view of the vanishing density of states at the Fermi level, an issue of particular interest is the influence of electron-electron interactions. González et al. ${ }^{3}$ performed renormalization-group calculations and showed that the suppression of screening of the long-range Coulomb interaction gives rise to deviations from conventional Fermi-liquid behavior. Lattice field theory simulations ${ }^{4}$ indicated a Coulomb driven second-order semimetal-to-insulator transition. Meng et al. ${ }^{5}$ performed extensive variational quantum Monte Carlo (QMC) simulations for the Hubbard model with varying cluster sizes and identified a spin-liquid phase between the semimetallic state characterized by massless Dirac fermions and an antiferromagnetically ordered Mott insulator. The onset of the long-range antiferromagnetic order was found to be consistent with previous QMC calculations for finite-size clusters. ${ }^{6,7}$ The Mott transition of the honeycomb lattice was also investigated ${ }^{8,9}$ within single-site dynamical mean field theory ${ }^{10}$ (DMFT). However, because of the small number of nearest neighbors, the neglect of spatial correlations in this system is questionable and gives rise to a significant overestimate of the range of the semimetallic behavior up to large values of the on-site Coulomb interaction. To account for nonlocal correlations in the honeycomb lattice, Wu et al. ${ }^{11}$ recently applied a cluster extension ${ }^{12}$ of DMFT (CDMFT) by using continuous-time QMC (Ref. 13) as an impurity solver. The transition between the semimetallic and insulating phases was found to occur at a considerably smaller critical Coulomb energy than within the single-site DMFT, and to be in good agreement with the variational QMC results by Meng et al. ${ }^{5}$

In the present work, we use finite-temperature exact diagonalization $^{14}$ (ED) in combination with cluster DMFT to investigate the two-dimensional Hubbard model on the honeycomb lattice for unit cells consisting of six sites. The focus is on the dynamical properties of the nonlocal selfenergy, which have not been studied before. Moreover, in view of the large size of this unit cell and the approximate nature of quantum impurity solvers, CDMFT results obtained within complementary schemes are clearly desirable. An important advantage of ED is the accessibility of large Coulomb energies and low temperatures, and the absence of sign problems. Also, in contrast to finite-size variational QMC, ED is applicable away from half-filling. On the other hand, due to the exponential growth of the Hilbert space, the number of levels representing the bath surrounding the cluster is severely limited. Here, we use 12 levels in total, that is, six impurity levels and six bath levels. Since these bath states are coupled indirectly via the on-site Coulomb repulsion within the six-atom cluster, the spacing between excitation energies is very small. Finite-size errors are thereby greatly reduced, even at low temperatures, so that self-energies and spectral functions can be evaluated reliably at rather low real frequencies.

The ED/CDMFT results discussed later reveal a continuous Mott transition in excellent correspondence with the variational QMC simulations by Meng et al. ${ }^{5}$ and with the QMC/CDMFT calculations by Wu et al. ${ }^{11}$ The critical on-site Coulomb energy is considerably smaller than that found in single-site DMFT calculations. ${ }^{8,9}$ Furthermore, short-range correlations included within CDMFT are shown to give rise to metallic and insulating contributions to the self-energy at the Dirac points in the Brillouin zone, where the former dominate at low Coulomb interactions and the latter increase essentially quadratically with the nearest-neighbor nonlocal self-energy component. These terms lead to the excitation gap above the Mott transition. Below the transition, they yield a narrow pseudogap. Thus, short-range correlations induced via on-site Coulomb repulsion give rise to deviations from Fermi-liquid behavior in some range below the critical interaction strength. Also, the effective-mass enhancement does not diverge at the Mott transition, but increases to a finite value. The opening of the pseudogap below the transition, and the variation of the effective mass with Coulomb energy, are qualitatively similar to analogous results obtained within cluster DMFT calculations for the square lattice. ${ }^{15-17}$

The outline of this paper is as follows: In the next section, we briefly outline the application of ED/CDMFT to the Hubbard model for the honeycomb lattice. Section III provides a discussion of the results. The summary is presented in Sec. IV. The focus in this work is on the paramagnetic semimetal-toinsulator transition. Spin-liquid and antiferromagnetic phases will be addressed in a subsequent publication. 


\section{CLUSTER ED/DMFT FOR THE HONEYCOMB LATTICE}

In this section, we discuss the combination of cluster DMFT with finite-temperature ED for the purpose of evaluating the effect of nonlocal Coulomb interactions on the honeycomb lattice. The Hubbard Hamiltonian is given by

$$
H=-t \sum_{\langle i j\rangle \sigma}\left(c_{i \sigma}^{+} c_{j \sigma}+\text { H.c. }\right)+U \sum_{i} n_{i \uparrow} n_{i \downarrow},
$$

where the sum in the first term includes only nearest neighbors, $t$ is the hopping matrix element, and $U$ is the on-site Coulomb repulsion. The band dispersion for the honeycomb lattice may be written as $\epsilon(\mathbf{k})= \pm t\left|1+e^{i k_{x} \sqrt{3}}+e^{i\left(k_{x} \sqrt{3}+k_{y} 3\right) / 2}\right|$. In the following, we define $t=1$ as an energy unit.

Let us divide the two-dimensional lattice into clusters consisting of six sites. Within the unit cell, the positions are specified as $\mathbf{a}_{1}=(0,0), \mathbf{a}_{2}=(1,0), \mathbf{a}_{3}=(\sqrt{3} / 2,3 / 2)$, $\mathbf{a}_{4}=(\sqrt{3}, 1), \mathbf{a}_{5}=(\sqrt{3}, 0)$, and $\mathbf{a}_{6}=(\sqrt{3} / 2,-1 / 2)$. The nearest-neighbor spacing is taken to be $a=1$. The supercell lattice vectors are then given by $A_{1 / 2}=(3 \sqrt{3} / 2, \pm 3 / 2)$. Within CDMFT, ${ }^{12}$ the interacting lattice Green's function in the cluster site basis is given by

$$
G_{i j}\left(i \omega_{n}\right)=\sum_{\mathbf{k}}\left[i \omega_{n}+\mu-t(\mathbf{k})-\Sigma\left(i \omega_{n}\right)\right]_{i j}^{-1},
$$

where $\omega_{n}=(2 n+1) \pi T$ are Matsubara frequencies and $\mu$ is the chemical potential. The $\mathbf{k}$ sum extends over the reduced Brillouin zone, $t(\mathbf{k})$ denotes the hopping matrix for the superlattice, and $\Sigma_{i j}\left(i \omega_{n}\right)$ represents the cluster self-energy matrix in the site representation. The diagonal elements of the symmetric matrix $G_{i j}$ are identical and there are three independent off-diagonal elements: $G_{12}=G_{16}, G_{13}=G_{15}$, and $G_{14}$. Because of these symmetry properties, it is convenient to go over to a diagonal "molecular orbital basis," in which the elements $G_{m}\left(i \omega_{n}\right)$ are given by

$$
\begin{gathered}
G_{1 / 2}=\left(G_{11}+2 G_{13}\right) \pm\left(G_{14}+2 G_{12}\right), \\
G_{3 / 4}=G_{5 / 6}=\left(G_{11}-G_{13}\right) \pm\left(G_{14}-G_{12}\right) .
\end{gathered}
$$

The self-energy matrix satisfies the same symmetry properties as $G$ and can therefore be diagonalized in the same fashion. These elements will be denoted as $\Sigma_{m}\left(i \omega_{n}\right)$. Later we will focus on the special case of half-filling. Since the density of states is then particle-hole symmetric with respect to $\omega=0, G_{i i}\left(i \omega_{n}\right)$ is purely imaginary. The same applies to $G_{13}$, whereas $G_{12}$ and $G_{14}$ are real, corresponding to odd density-of-states components. Thus, the diagonal molecular orbital components of $G$ satisfy $G_{2}=-G_{1}^{*}$ and $G_{4}=-G_{3}^{*}$. Figure 1 illustrates the uncorrelated density-of-states components in the diagonal basis, where $\rho_{m}(\omega)=-\frac{1}{\pi} \operatorname{Im} G_{m}(\omega)$ for $\Sigma=0$. The even and odd on-site and intersite components may be obtained by inverting Eq. (3).

A central feature of DMFT is that, to avoid double-counting of Coulomb interactions in the quantum impurity calculation, the self-energy must be removed from the small cluster in which correlations are treated explicitly. This removal yields the impurity Green's function

$$
G_{0}\left(i \omega_{n}\right)=\left[G\left(i \omega_{n}\right)^{-1}+\Sigma\left(i \omega_{n}\right)\right]^{-1} .
$$

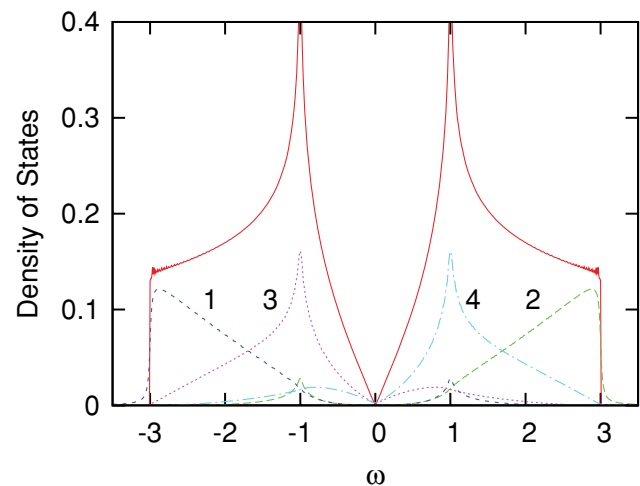

FIG. 1. (Color online) Total density of states $\rho(\omega)$ (solid curve) of the honeycomb lattice and cluster components $\rho_{m}(\omega)$ (dashed curves) within a diagonal molecular orbital basis. For clarity, these components are divided by $n_{c}=6$. Orbitals 3 and 4 are doubly degenerate. $\omega=0$ defines the Fermi energy for half-filling.

For the purpose of performing the ED calculation, we now project the diagonal components of $G_{0}\left(i \omega_{n}\right)$ onto those of a larger cluster consisting of six impurity levels and six bath levels, that is, $n_{s}=12$ is the total number of levels. Thus,

$$
\begin{aligned}
G_{0, m}\left(i \omega_{n}\right) & \approx G_{0, m}^{\mathrm{cl}}\left(i \omega_{n}\right) \\
& =\left(i \omega_{n}+\mu-\epsilon_{m}-\sum_{k=7}^{12} \frac{\left|V_{m k}\right|^{2}}{i \omega_{n}-\epsilon_{k}}\right)^{-1},
\end{aligned}
$$

where $\epsilon_{m}$ denotes impurity levels, $\epsilon_{k}$ denotes bath levels, and $V_{m k}$ denotes hybridization matrix elements. The incorporation of the impurity level $\epsilon_{m}$ in the fitting procedure yields a more accurate representation of $G_{0, m}\left(i \omega_{n}\right)$ than by projecting only onto bath levels.

Assuming independent baths for the diagonal cluster molecular orbitals, each component $G_{0, m}\left(i \omega_{n}\right)$ is fitted using three parameters: one impurity level $\epsilon_{m}$, a bath level $\epsilon_{k}$, and a hopping integral $V_{m k}$. To evaluate the finite-temperature interacting Green's function of the cluster, it is useful to transform the impurity orbitals back to the site representation in which the Coulomb interaction is diagonal. We denote this transformation by $\bar{T}$, where the matrix elements are given by

$$
\bar{T}_{i m}=\left(\begin{array}{rrrrrr}
\frac{1}{\sqrt{6}} & \frac{1}{\sqrt{6}} & 0 & \frac{1}{\sqrt{3}} & \frac{1}{\sqrt{3}} & 0 \\
\frac{1}{\sqrt{6}} & -\frac{1}{\sqrt{6}} & -\frac{1}{2} & \frac{1}{2 \sqrt{3}} & -\frac{1}{2 \sqrt{3}} & \frac{1}{2} \\
\frac{1}{\sqrt{6}} & \frac{1}{\sqrt{6}} & \frac{1}{2} & -\frac{1}{2 \sqrt{3}} & -\frac{1}{2 \sqrt{3}} & \frac{1}{2} \\
\frac{1}{\sqrt{6}} & -\frac{1}{\sqrt{6}} & 0 & -\frac{1}{\sqrt{3}} & \frac{1}{\sqrt{3}} & 0 \\
\frac{1}{\sqrt{6}} & \frac{1}{\sqrt{6}} & -\frac{1}{2} & -\frac{1}{2 \sqrt{3}} & -\frac{1}{2 \sqrt{3}} & -\frac{1}{2} \\
\frac{1}{\sqrt{6}} & -\frac{1}{\sqrt{6}} & \frac{1}{2} & \frac{1}{2 \sqrt{3}} & -\frac{1}{2 \sqrt{3}} & -\frac{1}{2}
\end{array}\right) .
$$

Thus, the diagonal $6 \times 6$ subblock of the cluster Hamiltonian, $h_{b}=\left(\epsilon_{k} \delta_{k k^{\prime}}\right)$, representing the bath levels remains unchanged, while the $6 \times 6$ impurity subblock becomes nondiagonal in 
the cluster site basis:

$$
h_{c}=\left(\begin{array}{llllll}
\epsilon & \tau & \tau^{\prime} & \tau^{\prime \prime} & \tau^{\prime} & \tau \\
\tau & \epsilon & \tau & \tau^{\prime} & \tau^{\prime \prime} & \tau^{\prime} \\
\tau^{\prime} & \tau & \epsilon & \tau & \tau^{\prime} & \tau^{\prime \prime} \\
\tau^{\prime \prime} & \tau^{\prime} & \tau & \epsilon & \tau & \tau^{\prime} \\
\tau^{\prime} & \tau^{\prime \prime} & \tau^{\prime} & \tau & \epsilon & \tau \\
\tau & \tau^{\prime} & \tau^{\prime \prime} & \tau^{\prime} & \tau & \epsilon
\end{array}\right),
$$

with

$$
\begin{gathered}
\epsilon=\left[\left(\epsilon_{1}+\epsilon_{2}\right)+2\left(\epsilon_{3}+\epsilon_{4}\right)\right] / 6, \\
\tau=\left[\left(\epsilon_{1}-\epsilon_{2}\right)-\left(\epsilon_{3}-\epsilon_{4}\right)\right] / 6, \\
\tau^{\prime}=\left[\left(\epsilon_{1}+\epsilon_{2}\right)-\left(\epsilon_{3}+\epsilon_{4}\right)\right] / 6, \\
\tau^{\prime \prime}=\left[\left(\epsilon_{1}-\epsilon_{2}\right)+2\left(\epsilon_{3}-\epsilon_{4}\right)\right] / 6 .
\end{gathered}
$$

We point out that the hopping element $t$ of the original lattice Hamiltonian does not appear since it is absorbed into $\tau$ via the molecular orbital cluster levels $\epsilon_{m}$, which are adjusted to fit $G_{0, m}\left(i \omega_{n}\right)$. The preceding procedure, therefore, includes not only hopping between cluster and bath. It also introduces four new parameters within the six-site cluster: the on-site level $\epsilon$, and up to third-neighbor hopping parameters: $\tau, \tau^{\prime}$, and $\tau^{\prime \prime}$. At half-filling, $\epsilon_{2}=-\epsilon_{1}$ and $\epsilon_{4}=-\epsilon_{3}$ for symmetry reasons, so that $\epsilon=\tau^{\prime}=0$. In this mixed site-molecular orbital basis, the hybridization matrix elements $V_{m k}$ between cluster and bath molecular orbitals introduced in Eq. (5) are transformed to new hybridization matrix elements between cluster sites $i$ and bath orbitals $k$. They are given by

$$
V_{i k}^{\prime}=(\bar{T} V)_{i k}=\sum_{m} \bar{T}_{i m} V_{m k}
$$

The single-particle part of the cluster Hamiltonian now reads

$$
h_{0}=\left(\begin{array}{ll}
h_{c} & V^{\prime} \\
V^{\prime t} & h_{b}
\end{array}\right) .
$$

Adding the on-site Coulomb interactions to this Hamiltonian, the nondiagonal interacting cluster Green's function at finite $T$ can be derived from the expression ${ }^{18,19}$

$$
\begin{aligned}
G_{i j}^{\mathrm{cl}}\left(i \omega_{n}\right)= & \frac{1}{Z} \sum_{\nu \mu} e^{-\beta E_{v}}\left(\frac{\left\langle\nu\left|c_{i \sigma}\right| \mu\right\rangle\left\langle\mu\left|c_{j \sigma}^{+}\right| \nu\right\rangle}{E_{\nu}-E_{\mu}+i \omega_{n}}\right. \\
& \left.+\frac{\left\langle\nu\left|c_{i \sigma}^{+}\right| \mu\right\rangle\left\langle\mu\left|c_{j \sigma}\right| \nu\right\rangle}{E_{\mu}-E_{\nu}+i \omega_{n}}\right),
\end{aligned}
$$

where $E_{v}$ and $|v\rangle$ denote the eigenvalues and eigenvectors of the Hamiltonian, $\beta=1 / T$, and $Z=\sum_{v} \exp \left(-\beta E_{v}\right)$ is the partition function. Further details concerning the evaluation of the cluster Green's function can be found in Ref. 20, where the analogous procedure is discussed for the square lattice. Since $G_{i j}^{\mathrm{cl}}$ satisfies the same symmetry properties as $G_{i j}$, it is diagonal within the molecular orbital basis, with elements $G_{m}^{\mathrm{cl}}$. The diagonal cluster self-energy components are then given by an expression analogous to Eq. (4):

$$
\Sigma_{m}^{\mathrm{cl}}\left(i \omega_{n}\right)=1 / G_{0, m}^{\mathrm{cl}}\left(i \omega_{n}\right)-1 / G_{m}^{\mathrm{cl}}\left(i \omega_{n}\right) .
$$

The important assumption in DMFT is now that this impurity cluster self-energy is a physically reasonable rep- resentation of the lattice self-energy. Thus,

$$
\Sigma_{m}\left(i \omega_{n}\right) \approx \Sigma_{m}^{\mathrm{cl}}\left(i \omega_{n}\right),
$$

where, at real frequencies, $\Sigma_{m}(\omega)$ is continuous whereas $\Sigma_{m}^{\mathrm{cl}}(\omega)$ is discrete.

In the next iteration step, these diagonal self-energy components are used as input in the lattice Green's function Eq. (2), which in the molecular orbital basis may be written as

$$
G_{m}\left(i \omega_{n}\right)=\sum_{\mathbf{k}}\left[i \omega_{n}+\mu-\bar{T} t(\mathbf{k}) \bar{T}^{-1}-\Sigma\left(i \omega_{n}\right)\right]_{m m}^{-1},
$$

where $\bar{T}$ is the transformation defined in Eq. (6). Note that $\bar{T} t(\mathbf{k}) \bar{T}^{-1}$ is not diagonal at general $\mathbf{k}$ points. As a result, all molecular orbital components of $\Sigma\left(i \omega_{n}\right)$ contribute to all components $G_{m}\left(i \omega_{n}\right)$. We also point out that, to get adequate resolution at low frequencies, because of the vanishing density of states, a sufficiently large number of $\mathbf{k}$ values near the Dirac points must be included in the Brillouin zone integration.

\section{RESULTS AND DISCUSSION}

Figure 2(a) shows the low-energy region of the interacting density of states for several Coulomb energies, at temperature $T=0.005$. These distributions are derived from an extrapolation of the local lattice Green's function $G_{11}\left(i \omega_{n}\right)$ to real frequencies. To illustrate the stability of this extrapolation,
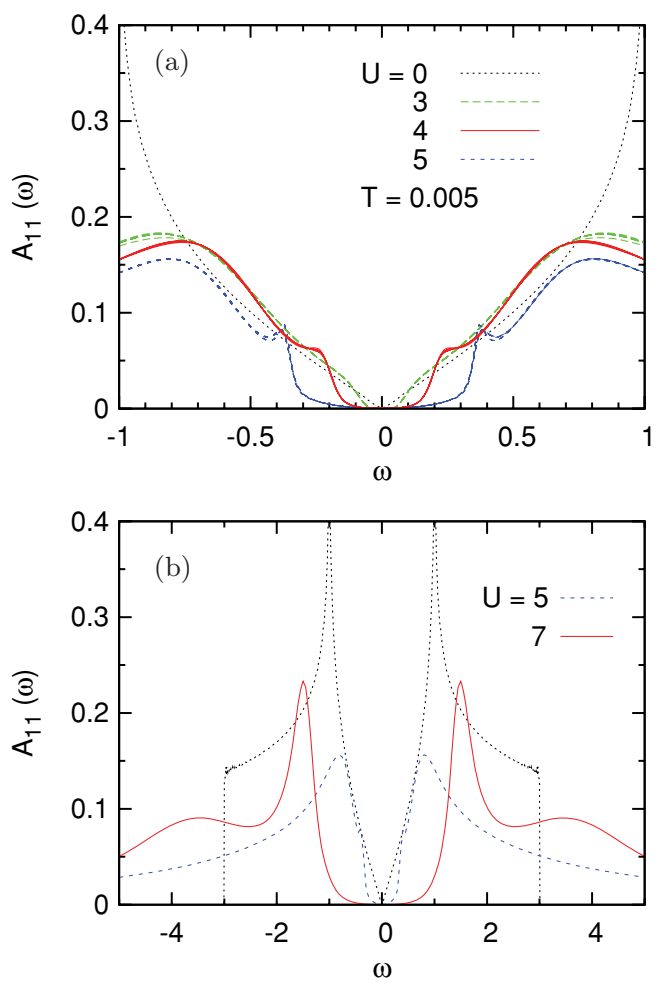

FIG. 2. (Color online) (a) Low-energy region of density of states $A_{11}(\omega)=-\frac{1}{\pi} \operatorname{Im} G_{11}(\omega)$ of the honeycomb lattice for several Coulomb energies at $T=0.005$. The noninteracting density of states is indicated by the black dotted curve. Between 50 and 200 Matsubara points are used to extrapolate the lattice Green's function to real frequencies. (b) Density of states over a wider energy range for $U=5$ and 7. 
at each value of $U$ several curves are plotted for 50 to 200 Matsubara points, with an additional small energy broadening of the order of $0.1 \omega^{2}$. (For $|\omega|>1$, the broadening is kept constant at 0.1.) At $U=3$, a tiny gap or pseudogap is seen, which is near the limit of what can be resolved within ED/DMFT. At $U=4$, a full gap of width $\Delta \approx 0.25$ has opened. Its width increases approximately to $\Delta \approx 0.6$ when the Coulomb energy is increased to $U=5$. This trend is consistent with the one found in Refs. 5 and 11. The variation of the gap over a wider range of $U$ is indicated in Fig. 3(a). The spectral distributions in Fig. 2 show that the van Hove singularity at $\omega= \pm 1$ is strongly broadened and its weight is shifted to lower energies. Above the transition, the Hubbard bands are difficult to resolve as long as $U$ is less than the bandwidth, but they become pronounced once $U>W$, as shown in Fig. 2(b) for $U=7$.

These results suggest that nonlocal correlations in the honeycomb lattice induce a paramagnetic semimetal-to-insulator Mott transition in the range $U=3-4$. Because of the continuous nature of the transition (see later), it is difficult to identify the precise value of the critical interaction. Nevertheless, our finding is consistent with the variational QMC simulations ${ }^{5}$ and the QMC/DMFT calculations, ${ }^{11}$ which yield $U_{c} \approx 3.6$. It is also in qualitative agreement with earlier finite-size cluster QMC simulations, which gave $U_{c} \approx 4.5$ (Ref. 6) and $U_{c} \approx 4-5$ (Ref. 7). On the other hand, all of these values are significantly lower than those obtained within single-site DMFT, which yields $U_{c} \approx 10-13 .{ }^{8,9}$ Moreover, in agreement with Refs. 5-7 and 11, we do not find any hysteresis behavior for increasing versus decreasing $U$, as shown in Fig. 3(b), for the double occupancy, indicating that the transition is continuous. In contrast, within local DMFT the transition was shown to be of first order. ${ }^{9}$ Figure 3(c) shows the on-site and intersite spin correlations, $\left\langle S_{1 z} S_{i z}\right\rangle$, for $i=1-4$. The on-site and second-neighbor components are positive, while the firstand third-neighbor components are negative, underlining the antiferromagnetic nature of the spin correlations.

One of the interesting effects of Coulomb interactions in multiorbital systems is the possibility of correlation-induced charge transfer between orbitals. As shown in Fig. 1, the six-site unit cell of the honeycomb lattice may be viewed as consisting of six molecular orbitals, which are split by an effective crystal field and therefore have different orbital occupancies. Figure 3(d) shows the variation of these occupancies with Coulomb energy. Evidently, there is little orbital polarization, a result that was also observed in CDMFT calculations for the square and triangular lattices. ${ }^{21}$ Moreover, the double occupancy, the spin correlations, and the orbital occupancies reveal no clear sign of a Mott transition in the region where the spectral distribution exhibits the opening of a gap.

To analyze the nature of the semimetal-to-insulator transition, it is therefore necessary to examine the nonlocal contributions to the self-energy. Figure 4 shows the four independent components of the cluster self-energy $\Sigma\left(i \omega_{n}\right)$ within the site basis, for Coulomb energies in the region of interest, $U=3-5$. For symmetry reasons, $\Sigma_{11}\left(i \omega_{n}\right)$ and $\Sigma_{13}\left(i \omega_{n}\right)$ are purely imaginary. They behave as $\sim i \omega_{n}$ at low frequencies. In contrast, $\Sigma_{12}\left(i \omega_{n}\right)$ and $\Sigma_{14}\left(i \omega_{n}\right)$ are real and approach a finite value in the limit $\omega_{n}=0$.
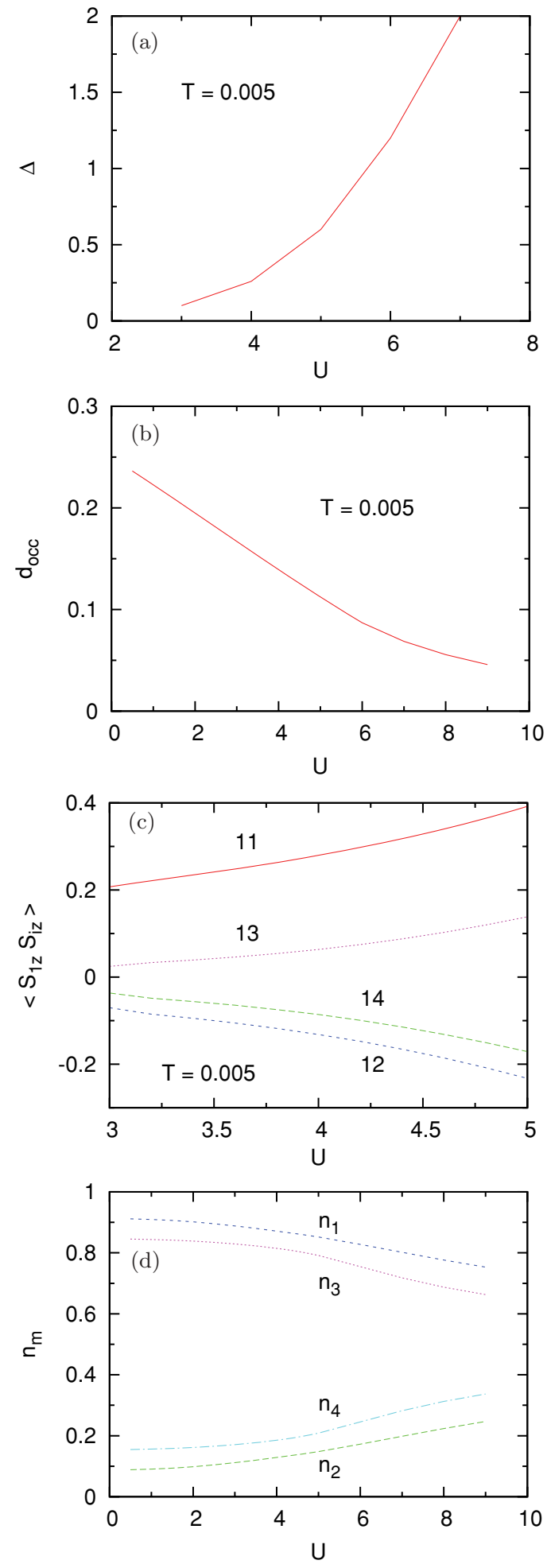

FIG. 3. (Color online) (a) Excitation gap $\Delta$, (b) average double occupancy $d_{\text {occ }}$, (c) local and nonlocal spin correlations $\left\langle S_{1 z} S_{i z}\right\rangle$, and (d) molecular orbital occupancies $n_{m}$ as functions of Coulomb energy for $T=0.005$. Orbitals 3 and 4 are doubly degenerate. There is no indication of hysteresis behavior in the critical region $U=3-4$.

In a seminal paper long before the synthesis of graphene, González et $\mathrm{al}^{3}{ }^{3}$ studied the influence of electron-electron interactions on the quasiparticle lifetime in a single layer of 

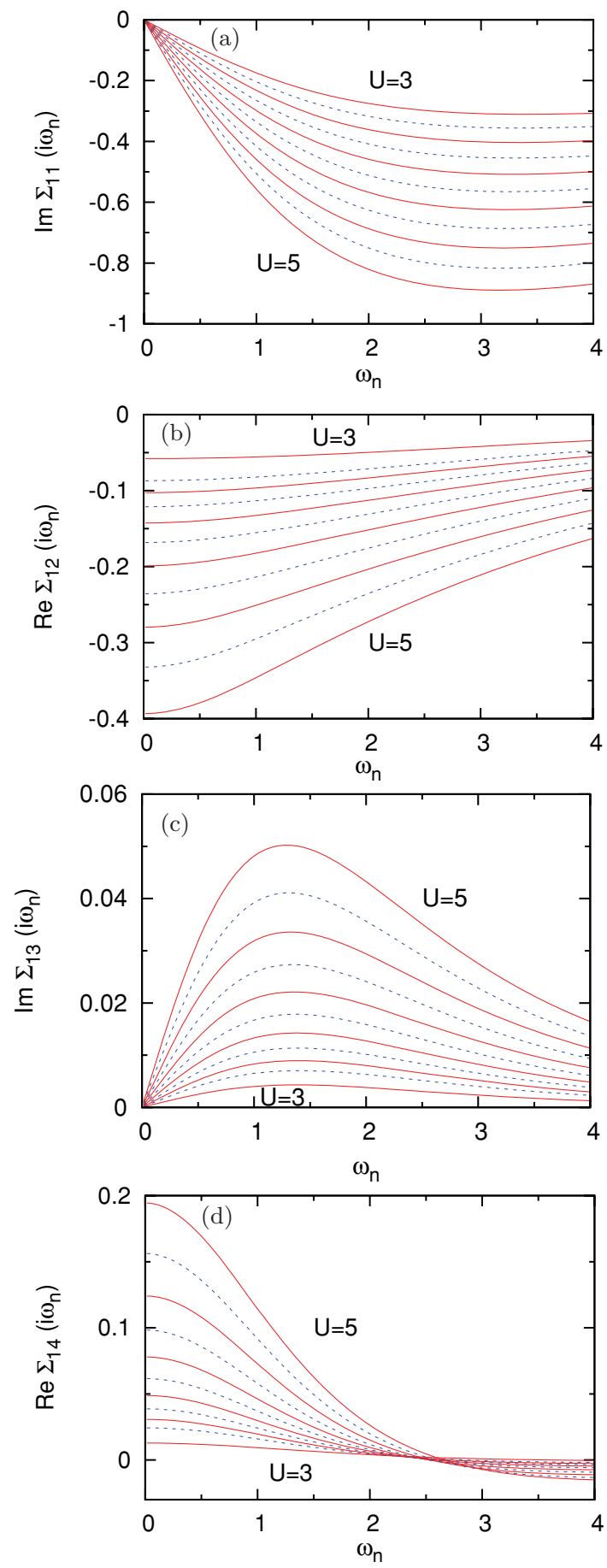

FIG. 4. (Color online) Self-energy components $\Sigma_{1 i}\left(i \omega_{n}\right)$, $i=1-4$, for the honeycomb lattice in cluster site representation as functions of Matsubara frequency for Coulomb energies $U=3-5$ in steps of $0.2 ; T=0.005$.

graphite. Taking into account the long-range nature of the Coulomb interaction, their renormalization-group calculations indicate that the suppression of electronic screening at low frequencies yields deviations from conventional Fermi-liquid behavior, with $\operatorname{Im} \Sigma(\omega)$ approximately linear in $\omega$ for $\omega=0.4-3.0 \mathrm{eV}$ (for $t=2.4 \mathrm{eV}$ ).

To determine possible non-Fermi-liquid contributions to the self-energy derived within the present ED/CDMFT approach, we have carefully searched for $\omega_{n} \ln \left(\omega_{n}\right)$ behavior in the imaginary components $\Sigma_{11}\left(i \omega_{n}\right)$ and $\Sigma_{13}\left(i \omega_{n}\right)$. Within the accuracy of our results, these functions do not indicate any such deviations and seem to be well proportional to $i \omega_{n}$ in the entire range $U=0-5$. Also, they do not indicate a finite limiting value for $\omega_{n} \rightarrow 0$, which would imply a finite lifetime for states near the Fermi energy. Thus, the non-Fermi-liquid properties obtained in Ref. 3 seem to be associated with the long-range part of the Coulomb repulsion, which is absent in the Hubbard model for purely on-site interactions. $^{22}$

We note, however, that to understand the spectral features of the quasiparticle density of states, it is not sufficient to study the self-energy components shown in Fig. 4. In particular, these isolated components do not provide any evidence for a Mott transition in the region $U=3-4$, where the density of states shown in Fig. 2 indicates the opening of a gap. To illustrate the smoothness of the self-energy components in this range of Coulomb energies, we show in Fig. 5(a) the slopes of $\operatorname{Im} \Sigma_{11}$ and $\operatorname{Im} \Sigma_{13}$, and the values of $\operatorname{Re} \Sigma_{12}$ and $\operatorname{Re} \Sigma_{14}$ in the low-frequency limit. Evidently, these individual components do not reveal the existence of the Mott transition seen in the density of states. This behavior differs qualitatively from the Hubbard model for the square lattice at half-filling, where at the metal-insulator transition the $(\pi, 0)$ component of the self-energy changes from $\sim i \omega_{n}$ to $\sim 1 / i \omega_{n}$ at small $\omega_{n}$,
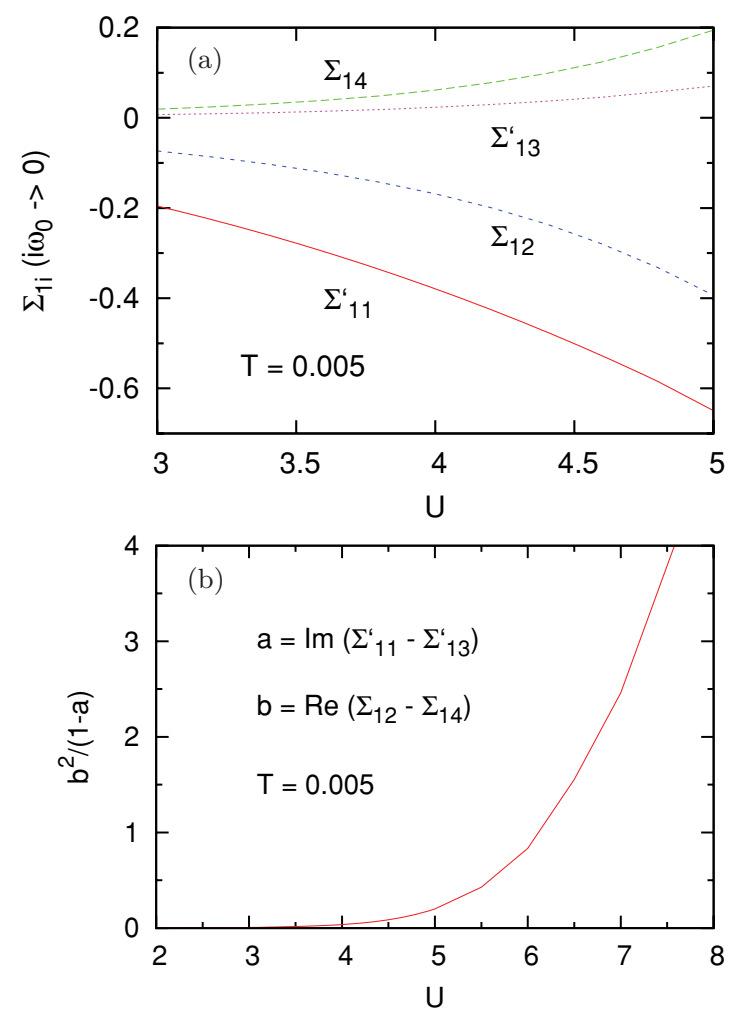

FIG. 5. (Color online) (a) Low-frequency limits of self-energy components: slopes of $\operatorname{Im} \Sigma_{11}\left(i \omega_{n}\right), \operatorname{Im} \Sigma_{13}\left(i \omega_{n}\right)$, and values of $\operatorname{Re} \Sigma_{12}\left(i \omega_{n}\right)$, Re $\Sigma_{14}\left(i \omega_{n}\right)$, as functions of $U$. (b) Amplitude $b^{2} /(1-a)$ of insulating contribution to $\Sigma\left(K, i \omega_{n}\right)$, Eq. (20), as a function of $U ; T=0.005$. 
and the real part of the $(0,0)$ and $(\pi, \pi)$ components exhibits a jump. ${ }^{16,17}$

The origin of this apparent discrepancy is the fact that, as pointed out earlier, the local interacting density of states depends in a highly nonlinear manner on all nonlocal self-energy components $\Sigma_{i j}$. This is evident from the expression for the lattice Green's function, Eq. (2), where the hopping matrix $t(\mathbf{k})$ and $\Sigma\left(i \omega_{n}\right)$ cannot be simultaneously diagonalized, as indicated also in Eq. (14). To account for this admixture of intersite self-energy elements, it is useful to examine the $6 \times 6$ cumulant matrix

$$
M\left(i \omega_{n}\right)=\left[i \omega_{n}-\Sigma\left(i \omega_{n}\right)\right]^{-1} .
$$

Since $M$ has the same symmetry properties as $\Sigma$, its nonlocal components are given by

$$
\begin{gathered}
M_{11}=\left[\left(M_{1}+M_{2}\right)+2\left(M_{3}+M_{4}\right)\right] / 6, \\
M_{12}=\left[\left(M_{1}-M_{2}\right)-\left(M_{3}-M_{4}\right)\right] / 6, \\
M_{13}=\left[\left(M_{1}+M_{2}\right)-\left(M_{3}+M_{4}\right)\right] / 6, \\
M_{14}=\left[\left(M_{1}-M_{2}\right)+2\left(M_{3}-M_{4}\right)\right] / 6,
\end{gathered}
$$

where the diagonal molecular orbital elements are

$$
M_{m}\left(i \omega_{n}\right)=\left[i \omega_{n}-\Sigma_{m}\left(i \omega_{n}\right)\right]^{-1} .
$$

The opening of the Mott gap takes place at the six $K$ points of the Brillouin zone. To analyze the behavior of the cumulant at these points, we make use of the periodization ${ }^{23}$

$$
M\left(\mathbf{k}, i \omega_{n}\right)=\frac{1}{6} \sum_{i j=1}^{6} e^{i \mathbf{k} \cdot\left(\mathbf{a}_{i}-\mathbf{a}_{j}\right)} M_{i j}\left(i \omega_{n}\right),
$$

where $\mathbf{a}_{i}$ are the positions within the six-site cluster. At $K=2 \pi(2 / 3 \sqrt{3}, 0)$ and $K^{\prime}=2 \pi(1 / 3 \sqrt{3}, 1 / 3)$, this expression simplifies to

$$
\begin{aligned}
M\left(K, i \omega_{n}\right) & =M_{11}\left(i \omega_{n}\right)-M_{13}\left(i \omega_{n}\right) \\
& =\left[M_{3}\left(i \omega_{n}\right)+M_{4}\left(i \omega_{n}\right)\right] / 2 .
\end{aligned}
$$

The self-energy at $K$ is therefore given by

$$
\begin{aligned}
\Sigma\left(K, i \omega_{n}\right) & =i \omega_{n}-M^{-1}\left(K, i \omega_{n}\right) \\
& \approx i \omega_{n} a+\frac{b^{2}}{i \omega_{n}(1-a)}, \quad \omega_{n} \rightarrow 0,
\end{aligned}
$$

where $a$ is the initial slope of $\operatorname{Im}\left[\Sigma_{11}\left(i \omega_{n}\right)-\Sigma_{13}\left(i \omega_{n}\right)\right]$ and $b$ is the low-frequency limit of $\operatorname{Re}\left[\Sigma_{12}\left(i \omega_{n}\right)-\Sigma_{14}\left(i \omega_{n}\right)\right]$. This self-energy is shown in Fig. 6 for various Coulomb energies. The preceding expression indicates that $\Sigma\left(K, i \omega_{n}\right)$ is imaginary as expected for particle-hole symmetry at the Dirac points. It consists of metallic $\left(\sim i \omega_{n}\right)$ and insulating $\left(\sim 1 / i \omega_{n}\right)$ contributions. The insulating term, which is responsible for the opening of the Mott gap, increases quadratically with $b=\operatorname{Re}\left(\Sigma_{12}-\Sigma_{14}\right)$. Thus, the semimetal-to-insulator transition is driven primarily by the nearest-neighbor component of the nonlocal self-energy, with a minor additional contribution due to the third-neighbor self-energy, and a weak renormalization related to the initial slope of $\operatorname{Im}\left[\Sigma_{11}\left(i \omega_{n}\right)-\Sigma_{13}\left(i \omega_{n}\right)\right]$. The variation of the amplitude $b^{2} /(1-a)$ of the insulating term with Coulomb energy is depicted in Fig. 5(b). The
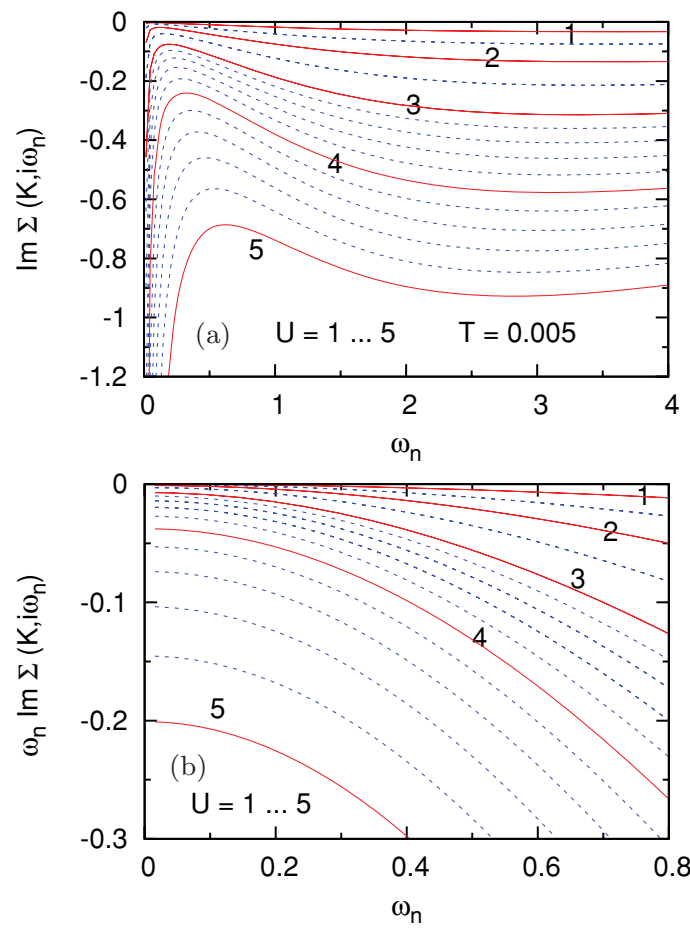

FIG. 6. (Color online) (a) Imaginary part of self-energy at $K$, Eq. (20), as a function of Matsubara frequency, for $U=1-5$ at $T=0.005$. (b) $\omega_{n} \operatorname{Im} \Sigma\left(K, i \omega_{n}\right)$, demonstrating the range of the insulating part of the self-energy at Dirac points $K$. Solid red curves denote integer values of $U$, dashed blue curves denote intermediate values.

comparison with Fig. 3(a) demonstrates that the excitation gap $\Delta$ roughly tracks the amplitude of this term.

According to the results shown in Fig. $4, a \approx-0.2, \ldots$, -0.6 and $b \approx-0.05, \ldots,-0.6$ in the range $U=3-5$. Thus at $U=3$, the amplitude of the insulating term is about $10^{2}$ times smaller than at $U=5$. Nevertheless, this small contribution is responsible for the pseudogap below the Mott transition, indicating the breakdown of Fermi-liquid behavior in the metallic phase. For $U<2$, we find $|b|<\omega_{0}$, so that the pseudogap can no longer be resolved within the accuracy of ED. A similar pseudogap induced by short-range correlations at half-filling was observed below the Mott transition in the Hubbard model for the square lattice. ${ }^{15-17}$ Neglecting the small insulating term of $\Sigma\left(K, i \omega_{n}\right)$ sufficiently far below the transition, the effective-mass enhancement of the quasiparticle bands near the Dirac points is given by $m^{*} / m=1-a=$ $1.0, \ldots, 1.2$ for $U=0-3$, that is, it does not diverge at the Mott transition, in contrast to results derived within single-site DMFT. This finding is also consistent with the behavior seen on the square lattice. ${ }^{16}$

We close this section by commenting briefly on the relevance of the results discussed earlier for graphene. There exists at present considerable debate on the role of screening and on the magnitude of the short-range Coulomb repulsion in this material. $^{2}$ In the case of a benzene molecule, the on-site Hubbard interaction was estimated to be $U \approx 17 \mathrm{eV}{ }^{24}$ In contrast, in polyacetylene this value is reduced to about $U \approx 10 \mathrm{eV}$, with a hopping interaction $t=2.5 \mathrm{eV} .^{25}$ The ratio $U / t \approx 4$ would then be slightly larger than the critical Coulomb interaction 
found here and in Refs. 5 and 11 . On the other hand, firstprinciples and mean field Hubbard model calculations ${ }^{26}$ yield ratios $U / t \approx 1, \ldots, 2$, depending on the exchange-correlation functional used in the density-functional calculation. There is clearly a great need for further theoretical and experimental work to investigate to what extent a purely on-site Hubbard interaction is adequate for graphene, and to determine its realistic magnitude.

\section{SUMMARY}

The influence of on-site Coulomb interactions on the electronic properties of the honeycomb lattice has been investigated within cluster dynamical mean field theory combined with exact diagonalization. The interacting density of states exhibits the opening of a Mott gap in the region $U=3-4$, which is caused by a change of the self-energy at the Dirac points of the Brillouin zone from metallicto-insulating behavior. This transition is in good agreement with finite-size extrapolations of variational QMC simulations and with continuous-time QMC calculations based on cluster
DMFT. As a result of short-range fluctuations, the critical Coulomb energy is significantly smaller than in single-site DMFT calculations. Also, a narrow pseudogap is found close to the Mott transition. Finally, the effective mass shows a moderate enhancement at finite $U$, but it does not diverge at the transition.

The consistency between the ED and QMC calculations for the honeycomb lattice, including the variation of the Mott gap with on-site Coulomb repulsion, suggests that, as long as the overall size of the Hilbert space is sufficiently large, yielding small enough level spacing, the use of only one bath level per impurity orbital can be adequate. This situation differs from the one for fewer sites or orbitals, where more bath levels per impurity level must be included to achieve sufficiently large Hilbert spaces.

\section{ACKNOWLEDGMENTS}

I would like to thank Hiroshi Ishida for valuable discussions. The ED/CDMFT calculations were carried out on the Jülich Juropa computer.
${ }^{1}$ K. S. Novoselov, A. K. Geim, S. V. Morosov, D. Jiang, Y. Zhang, S. V. Dubonos, I. V. Gregorieva, and A. A. Firsov, Science 306, 666 (2004).

${ }^{2}$ A. H. Castro Neto, F. Guinea, N. M. R. Peres, K. S. Novoselov, and A. K. Geim, Rev. Mod. Phys. 81, 109 (2009).

${ }^{3}$ J. González, F. Guinea, and M. A. H. Vozmediano, Phys. Rev. Lett. 77, 3589 (1996).

${ }^{4}$ J. E. Drut and T. A. Lähde, Phys. Rev. Lett. 102, 026802 (2009); Phys. Rev. B 79, 165425 (2009).

${ }^{5}$ Z. Y. Meng, T. C. Lang, S. Wessel, F. F. Assaad, and A. Muramatsu, Nature (London) 464, 847 (2010).

${ }^{6}$ S. Sorella and E. Tosatti, Europhys. Lett. 19, 699 (1992).

${ }^{7}$ Th. Paiva, R. T. Scalettar, W. Zheng, R. R. P. Singh, and J. Oitmaa, Phys. Rev. B 72, 085123 (2005).

${ }^{8}$ S. A. Jafari, Eur. Phys. J. B 68, 537 (2009).

${ }^{9}$ M. T. Tran and K. Kuroki, Phys. Rev. B 79, 125125 (2009).

${ }^{10}$ A. Georges, G. Kotliar, W. Krauth, and M. J. Rozenberg, Rev. Mod. Phys. 68, 13 (1996).

${ }^{11}$ W. Wu, Y.-H. Chen, H.-Sh. Tao, N.-H. Tong, and W.-M. Liu, Phys. Rev. B 82, 245102 (2010).

${ }^{12}$ G. Kotliar, S. Y. Savrasov, G. Palsson, and G. Biroli, Phys. Rev. Lett. 87, 186401 (2001).

${ }^{13}$ A. N. Rubtsov, V. V. Savkin, and A. I. Lichtenstein, Phys. Rev. B 72, 035122 (2005).

${ }^{14}$ M. Caffarel and W. Krauth, Phys. Rev. Lett. 72, 1545 (1994).
${ }^{15}$ B. Kyung, S. S. Kancharla, D. Sénéchal, A.-M. S. Tremblay, M. Civelli, and G. Kotliar, Phys. Rev. B 73, 165114 (2006).

${ }^{16}$ Y. Z. Zhang and M. Imada, Phys. Rev. B 76, 045108 (2007).

${ }^{17}$ H. Park, K. Haule, and G. Kotliar, Phys. Rev. Lett. 101, 186403 (2008).

${ }^{18}$ C. A. Perroni, H. Ishida, and A. Liebsch, Phys. Rev. B 75, 045125 (2007).

${ }^{19}$ M. Capone, L. de' Medici, and A. Georges, Phys. Rev. B 76, 245116 (2007).

${ }^{20}$ A. Liebsch and N.-H. Tong, Phys. Rev. B 80, 165126 (2009).

${ }^{21}$ A. Liebsch, H. Ishida, and J. Merino, Phys. Rev. B 78, 165123 (2008).

${ }^{22}$ F. Guinea, M. P. López-Sancho, and M. A. H. Vozmediano, in Carbon-based Magnetism: An Overview of the Magnetism of Metal Free Carbon-based Compounds and Materials, edited by T. Makarova and F. Palacio (Elsevier, Amsterdam, 2005), pp. 353-370.

${ }^{23}$ O. Parcollet, G. Biroli, and G. Kotliar, Phys. Rev. Lett. 92, 226402 (2004).

${ }^{24}$ R. G. Parr, D. P. Craig, and I. G. Ross, J. Chem. Phys. 18, 1561 (1950).

${ }^{25}$ D. Baeriswyl, D. K. Campbell, and S. Mazumdar, Phys. Rev. Lett. 56, 1509 (1986).

${ }^{26}$ L. Pisani, J. A. Chan, B. Montanari, and N. M. Harrison, Phys. Rev. B 75, 064418 (2007). 